\title{
WHY IT'S TIME TO SAY GOODBYE TO STAVUDINE ... EVERYWHERE
}

\author{
Isabelle Andrieux-Meyer, ${ }^{1} M D$ \\ Polly Clayden ${ }^{2}$ \\ Simon Collins ${ }^{2}$ \\ Nathan Geffen ${ }^{3}$ \\ Eric Goemaere, ${ }^{4} M D, D T M H, P h D$ \\ Mark Harrington ${ }^{5}$ \\ Sharonann Lynch ${ }^{1}$ \\ Tido von Schoen-Angerer, ${ }^{1} M D, M S c$ \\ Tracy Swan ${ }^{5}$ \\ ${ }^{1}$ Médecins Sans Frontières Access Campaign \\ ${ }^{2} \mathrm{HIV}$ i-Base \\ ${ }^{3}$ Treatment Action Campaign \\ ${ }^{4}$ Médecins Sans Frontières, South Africa \\ ${ }^{5}$ Treatment Action Group
}

Editor's note: The previous issue of the SAJHIV (December 2011) carried an Opinion piece by Innes, Cotton and Venter regarding the potential value of low-dose of stavudine ( $20 \mathrm{mg}$ twice a day). They suggested that reduced dosing of stavudine may lead to levels of viral suppression comparable with those achieved with stavudine $30 \mathrm{mg}$ bd but with a lower risk of toxicity and sideeffects, and at a fraction of the cost of tenofovir. The Opinion was related to a larger proposal, led by Venter, to conduct a head-to-head trial comparing low-dose stavudine with tenofovir (both in a regimen including lamivudine and efavirenz) on viral suppression and other treatment outcomes over 24 months. There has been considerable debate regarding the advantages and disadvantages of low-dose stavudine, and in turn the value of any such trial. Here the debate continues with a commentary by Isabelle Andrieux-Meyer et al. and a rebuttal by Venter and colleagues.

We read with interest an opinion piece by Innes et $a .^{1}$ ' in the previous issue of the journal, regarding the potential value of low-dose stavudine (20 mg twice daily). The article focused on stavudine use in paediatrics (where there are fewer approved antiretrovirals compared with adults, although there will be greater choice in the near future, as the US Food and Drug Administration (FDA) has recently approved tenofovir for 2 - 12-year-olds, and other regulatory agencies are expected to follow suit). In the article, the authors used the situation with children to argue for a proposal, led by Venter, to conduct a head-to-head non-inferiority study in adults comparing low-dose stavudine with tenofovir (both in a regimen including lamivudine and efavirenz) with a 48-week virological endpoint and other treatment outcomes over 96 weeks. * We have serious concerns about this proposed trial, for the following reasons:

1. Stavudine is more toxic than tenofovir, and for this reason it is an inferior treatment option. The proposed trial aims to establish virological non-inferiority, which is a moot point, given the severe adverse events associated with stavudine. Considerable evidence supports the use of tenofovir over stavudine; regulatory bodies and the World Health Organization (WHO) have turned away from the drug. In 2004, stavudine was removed from the list of preferred first-line antiretroviral drugs recommended by the US Department of Health and Human Services (DHHS). ${ }^{2}$ Starting in 2006, the WHO recommended that countries start moving away from stavudine, and in 2009 recommended that the drug be phased out in first-line antiretroviral therapy (ART) programmes. ${ }^{3}$ Earlier this year, the European Medicines Agency (EMA) revised the indication for stavudine, noting that '... the use of the medicine should be severely restricted in both adults and children ... Prescribers are reminded of the severe side effects seen with Zerit [stavudine] and should only use the medicine when other appropriate treatments are not available. Patients being treated with Zerit should be assessed frequently and switched to appropriate alternatives as soon as possible.' ${ }^{\prime 4}$

Médecins Sans Frontières (MSF)/Doctors Without Borders have provided further compelling evidence of stavudine's toxicity in an operational setting. In a Lesotho cohort, the authors found that '... for patients on stavudine, the risk of a toxicity-driven regimen switch was almost six times higher than tenofovir'.5 The high incidence of adverse events among patients on stavudinecontaining first-line regimens has also been documented in a larger prospective study in South Africa. ${ }^{6}$ In that study $30 \%$ of patients had to switch from stavudine-based to non-stavudinebased regimens within 3 years.

For good reasons, tenofovir has become the gold standard for today's first-line antiretroviral therapy. Its introduction in developing countries is an important step towards bringing treatment in poor countries in line with rich ones. As the WHO and all countries are phasing out stavudine, this study will send a confusing message, and it may slow down this transition while countries wait for the results.

${ }^{*}$ A randomised, double-blind study to demonstrate non-inferiority of stavudine (20 mg BID) compared with tenofovir (300 mg QD) c0-administered with lamivudine and efavirenz in antiretroviral-naive patients over 96 weeks. If funded and approved, the trial is anticipated to start in 2012. 
There is no prospect that stavudine $20 \mathrm{mg}$ is a better option than tenofovir. The stavudine parallel track programme, in which over 10000 patients were randomised to receive 40 (30) mg or 20 (15) mg between October 1992 and February 1994, showed a higher incidence of neuropathy in the high-dose arm (21\%). Nonetheless, the incidence of neuropathy observed in the lower-dose arm was also unacceptably high (15\%).

Of particular importance in low- to middle-income countries where tuberculosis (TB) is prevalent among HIV-positive people, who are also receiving stavudine-containing regimens - a South African study looked at the risk of stavudine substitution for toxicities in 7066 patients receiving ongoing TB treatment at ART initiation; concurrent initiation of TB treatment and ART and incident TB treatment after ART initiation. The study found people receiving ongoing and concurrent $T B$ treatment to be at increased risk of toxicity leading to stavudine substitution, irrespective of stavudine dose (30 and $40 \mathrm{mg}$ ). For ongoing TB treatment, adjusted hazard ratio (aHR) was 3.18 (95\% confidence interval (CI) 1.82 - 5.56) in the first 2 months of ART; for concurrent TB treatment, aHR was $6.60(95 \% \mathrm{Cl} 3.03-14.37)$ in the first 2 months of ART.

The stavudine $20 \mathrm{mg}$ study is not being proposed in any developed country. Instead it is planned to include only middle- and lowerincome developing countries. Patients enrolling in this trial risk being randomised to receive treatment that may be less effective and is more toxic than the current standard of care. There is therefore no good reason why a properly informed patient should want to enrol in this study.

2. The poor tolerability of stavudine limits therapeutic durability. A person has the best chance of successful treatment with their first-line regimen, making it critical that the medicines are as tolerable as possible. A tolerable first-line regimen enhances therapeutic durability by helping people adhere to treatment, and delays their need to switch to more costly second-line regimens, which are complicated for patients, for health workers and from an operational standpoint.

3. Stavudine's side-effects cut into stavudine's savings on cost. A study published by MSF shows that inpatient care and essential drug costs were higher for people on stavudine than for those on tenofovir in a cohort in rural Lesotho. According to MSF's cost-effectiveness study of switching from stavudine or zidovudine- to tenofovir-based first-line regimens in Lesotho, the tenofovir-containing regimen generated higher life years and quality-adjusted life years than zidovudine or stavudine-based treatment. ${ }^{8}$ As the costs of tenofovir and especially efavirenz drop, the cost benefit to patients and to health systems will become clearer. Since the study was completed, the global best price of efavirenz - which partly drives tenofovir costs - has almost halved (US\$97 per patient year in 2009 to $\$ 52$ today).

4. Stavudine can compromise second-line options. When someone does fail their first-line regimen, the longer they remain on stavudine - which is likely in a context with limited access to viral load monitoring - the more their second-line options are compromised. Unlike stavudine, tenofovir does not confer thymidine analogue mutations (TAMs); people taking tenofovir can stay on a failing regimen much longer without compromising efficacy of zidovudine and therefore second-line therapy.
5. Stavudine's long-term toxicity question will not be answered by this trial. The proposed $20 \mathrm{mg}$ stavudine dose might be acceptable in a short-term 48- or even 96-week virological endpoint study (although Bristol-Myers Squibb studied and rejected $20 \mathrm{mg} \mathrm{bd}$ ). But, because mitrochondrial toxicity is both dose and time dependent, many of stavudine's most serious sideeffects (such as peripheral neuropathy and lipoatrophy) would not necessarily emerge until after such a study was completed. This study does not include monitoring of surrogate markers for mitochondrial toxicity, so it cannot shed light on the incidence of this serious adverse event.

Recently published longer-term Cambodian data on rates of severe stavudine-associated toxicity show $7 \%$ of people to have neuropathy within the first year; by year 3 the cumulative incidence was $16.6 \%$, and it rose to $19.0 \%$ at year 6 . The cumulative incidence of lipoatrophy was $56 \%$ by year 3 and $72 \%$ by year 6 . Stavudine use significantly increased the risk for lactic acidosis among people on concurrent TB treatment; the aHR was $8.6(95 \% \mathrm{Cl} 2.7-27.5) .^{10}$

The investigators have agreed that this important question about longer-term toxicity will not be answered in the trial, raising the serious issue that the trial will not be able to answer the primary policy question which drives it - whether long-term $20 \mathrm{mg}$ stavudine twice daily is as good as once-daily tenofovir in first-line ART regimens for use in public health programmes in resourcelimited settings.

6. Stavudine must be taken twice a day, compared with tenofovir's once-daily dosing. A twice-daily dosing regimen (as with stavudine $20 \mathrm{mg}$ ) does not have the simplicity of a oncedaily fixed-dose combination (as with tenofovir). People are more likely to adhere to simpler regimens and therefore are more likely to have better treatment outcomes, as well as stave off resistance that requires more complex and expensive second-line regimens.

\section{A tenofovir-based regimen is recommended for HIV/} hepatitis B (HBV) coinfection, because stavudine has no activity against $\mathrm{HBV}$ and resistance to lamivudine is inevitable. While HIV/HBV co-infection is an exclusion criterion for this trial, it may encourage persistent use of a suboptimal regimen for HIV/HBV co-infected people. Screening for HBV is not routinely performed before initiation of ART in most resource-limited settings, yet $\mathrm{HBV}$ is endemic. For example, in South Africa an estimated 5\% of HIV-positive people are HBV coinfected (Dr Mark Sonderup, personal communication). Giving a stavudine/lamivudine-based regimen to HIV/HBV co-infected people will create lamivudine-resistant HBV in this population ( $90 \%$ at 4 years). ${ }^{9}$ Continuing lamivudine in the context of HBV drug resistance may lead to hepatitis flares; these flares can cause serious liver damage, and are potentially life-threatening. Researchers are also concerned about the transmission of drugresistant $H B V$ that may not be preventable by currently available $H B V$ vaccines, a potential public health catastrophe.

8. Stavudine-related cost savings may become irrelevant by the trial's end. The rationale for this trial is to lower treatment costs, as stavudine is currently cheaper than its alternatives. However, the price of alternatives, notably tenofovir, has come down dramatically in the last several years, and is expected to decrease further as demand increases. According to MSF's annual ARV pricing report, tenofovir is now cheaper than zidovudine, with 
the price of single-drug tenofovir having decreased by $52 \%$ from 2008 to 2011, and the price of the triple fixed-dose combination of tenofovir, lamivudine and efavirenz having decreased by $53 \%$ to US\$173 per person per year over that same time period. ${ }^{10}$ The price of the double FDC TDF/3TC co-packed with EFV is $\$ 143$ per person per year. Furthermore, the Clinton Health Access Initiative (CHAI) is currently working on the in the reformulation of tenofovir, with the goal of increasing bioavailability, hence reducing the required active pharmaceutical ingredient and in turn the cost.

Because the stavudine $20 \mathrm{mg} 96$-week efficacy trial is expected to be completed at the earliest by $2014-2015$, and would need to be followed by a larger, longer (perhaps 5-year) field effectiveness trial to determine longer-term tolerability, the drug may not be available for use at the new dose until possibly even 2020. It is therefore likely to take 9 years from now for there to be enough evidence that $20 \mathrm{mg}$ stavudine is safe and non-inferior to tenofovir, and could be used to replace tenofovir in first-line regimens.

If current price trends continue, it is likely the anticipated cost savings associated with stavudine could be overtaken by expected further price reductions for tenofovir and other components of the first-line regimen, by the time stavudine $20 \mathrm{mg}$ would be ready for use. It is worth noting that a three-drug one-pill-once-aday regimen containing efavirenz and tenofovir is now priced at roughly half what stavudine-based Triomune cost when it was first introduced a decade ago.

Further, even greater potential savings could be achieved if the tenofovir prodrug GS 7340, now in phase II by Gilead Sciences, is approved at a low milligram dose. Results will be available within a similar time frame to those from the 96 -week stavudine $20 \mathrm{mg}$ trial. A recent announcement by Gilead of an agreement with Tibotec to develop a fixed-dose combination of darunavir, emtricitabine, GS 7340 and cobicistat with 'less than one tenth of the amount of the $300 \mathrm{mg}$ of tenofovir disoproxil fumarate contained in Viread and Truvada' suggests that this is feasible. ${ }^{11}$ Chimerix Inc. also has a promising tenofovir pro-drug in development, CMX-157.

0ther drugs in late-stage development such as the integrase inhibitor dolutegravir (50 mg once daily) also offer potential savings on manufacturing and could end up being cheaper than stavudine $20 \mathrm{mg}$ by the time it would become available.

9. Stavudine has low acceptability in the community. Finally, and most importantly, the continued use of stavudine and the proposed trial has raised opposition from people directly affected by its continued use. As an example, the Malawi Network of People Living with HIV/AIDS (MANET+) recently held a press briefing, as the slow pace for phasing out current use of this drug in their country concerns them. Despite the funding crisis, the Malawi government has a priority for this to be completed by June $2012 .{ }^{12}$

It is unclear why the Bill and Melinda Gates Foundation - who are in discussion with the investigators about funding the proposal consider this study to be a priority. It seems an aberration in an otherwise carefully considered strategy for supporting research into the optimisation of ART for resource-limited settings. This includes the ENCORE 1 study of low-dose efavirenz, the reformulation of tenofovir to increase its bioavailability (working with (HAI), and the development of innovative potentially longacting formulations.

For the reasons outlined above, research and the resources it requires, as well as activist pressure, should focus on increasing access to safer cost-saving alternatives to stavudine, not on seeking a comeback for a drug virtually abandoned in wealthy countries.

\section{Please address all correspondence to:}

sharonann.lynch@msf.org

polly.clayden@i-base.org.uk

nathangeffen@gmail.com

markhar@gmail.com

\section{REFERENCES}

1. Innes S, Cotton M, Venter F. Why should we still care about the stavudine dose? Southern African Journal of HIV Medicine 201;12(4):14-15.

2. Panel on Clinical Practices for Treatment of HIV Infection. Guidelines for the use of antiretroviral agents in HIV-1-infected adults and adolescents. Department of Health and Human Services (DHHS), 2004. http://aidsinfo.nih.gov/ContentFiles/AdultandAdolescentGL10292004002.pdf (accessed 20 February 2012).

3. World Health Organization. WHO Rapid Advice: Antiretroviral therapy for HIV infection in adults and adolescents. WH0, 2009. http://www.who.int/hiv/pub/arv/rapid_advice_art.pdf (accessed 20 February 2012).

4. European Medicines Agency. Questions and answers on the review of Zerit (stavudine): Outcome of a renewal procedure. European Medicines Agency, 17 February 2011. http://www.ema.europa. eu/docs/en_GB/document_library/Medicine_QA/human/000110/WC500102227.pdf (accessed 20 February 2012).

5. Bygrave $\mathrm{H}$, Ford N, van Cutsem $\mathrm{G}$, et al. Implementing a tenofovir-based first-line regimen in rural Lesotho: clinical outcomes and toxicities after two years. J Acquir Immune Defic Syndr 2011;56(3):e75-78 [http://dx.doi.org/10.1097/QAl.0b013e3182097505].

6. Menezes C, Maskew M, Sanne I, Crowther N, Raal F. A longitudinal study of stavudine-associated toxicities in a large cohort of South African HIV infected subjects. BMC Infect Dis 2011;11:244 [http://dx.doi.org/10.1186/1471-2334-11-244].

7. Anderson R, Dunkle L, Smaldone L, et al. Design and implementation of the stavudine parallel track programme. J Infect Dis 1995;171:118-122. [http://dx.doi.org/10.1093/infdis/171.Supplement_2. S118].

8. Jouquet $G$, Bygrave $H$, Kranzer $K$, et al. Cost and cost-effectiveness of switching from d4T or AZT to a TDF-based first-line regimen in a resource limited setting in rural Lesotho. J Acquir Immune Defic Syndr 2011;58:e68-e74 [http://dx.doi.org/10.1097/QAl.0b013e31822a9f8d].

9. Benhamou $Y$, Bochet $M$, Thibault $V$, et al. Long-term incidence of hepatitis B virus resistance to Iamivudine in human immunodeficiency virus-infected patients. Hepatology 1999;30:1302-1306. [http://dx.doi.org/10.1002/hep.510300525].

10. Médecins Sans Frontières. Untangling the Web of Antiretroviral Price Reductions. 14th ed. Médecins Sans Frontières Campaign for Access to Essential Medicines, July 2011. http://www. doctorswithoutborders.org/publications/article.cfm?id=5448\&cat=special-report (accessed 20 February 2012).

11. Gilead. Gilead Sciences Finalizes Agreement with Tibotec Pharmaceuticals to Develop and Commercialize a Single-Tablet Regimen of Prezista(R) with Emtriva(R), GS 7340 and Cobicistat. Gilead Press Release. http://www.gilead.com/pr_1630785 (accessed 20 February 2012).

12. Nkhoma P. Manet+ wants ARV d4T phased out The Daily Times, Malawi, 30 January 2012. http:// www.bnltimes.com/index.php/daily-times/headlines/national/4079-manet-wants-arv-d4tphased-ou (accessed 20 February 2012). 